\title{
A novel two-phase approach for solving the multi-compartment vehicle routing problem with a heterogeneous fleet of vehicles: a case study on fuel delivery
}

\author{
Wasana Chowmali $^{\mathbf{a}^{*}}$ and Seekharin Sukto ${ }^{\mathrm{a}}$
}

\begin{abstract}
${ }^{a}$ Department of Industrial Engineering, Faculty of Engineering, Khon Kaen University, Khon Kaen, 40002, Thailand
A B S T R A C T

Distribution of goods is one of the main issues that directly affect the performance of the companies since efficient distribution of goods saves energy costs and also leads to reduced environmental impact. The multi-compartment vehicle routing problem (MCVRP) with a heterogeneous fleet of vehicles is encountered when dealing with this situation in many practical cases. This paper is motivated by the fuel delivery problem where the main objective of this research is to minimize the total driving distance using a minimum number of vehicles. Based on a case study of twenty petrol stations in northeastern Thailand, a novel two-phase heuristic, which is a variant of the Fisher and Jaikumar Algorithm (FJA), is proposed. The study first formulates an MCVRP model and then a mixed-integer linear programming (MILP) model is formulated for selecting the numbers and types of vehicles. A new clustering-based model is also developed in order to select the seed nodes and all customer nodes are considered as candidate seed nodes. The new Generalized Assignment Problem model (GAP model) is formulated to allocate the customers into each cluster. Finally, based on the traveling salesman problem (TSP), each cluster is solved in order to minimize the total driving distance. Numerical results show that the proposed heuristic is effective for solving the proposed model. The proposed algorithm can be used to minimize the total driving distance and the number of vehicles of the distribution network for fuel delivery.
\end{abstract}

\section{Introduction}

There are a lot of real-world problems which are very hard to tackle using exact methods. The Vehicle Routing Problem (VRP), which is famous as an NP-hard problem, is a well-known problem in operations research and combinatorial optimization (Chokanat et al., 2019; Wichapa \& Khokhajaikiat, 2018). Much attention of researchers has been devoted on the development of the characteristics of the problem and assumptions, leading to an enormous number of VRPs and variants, as well as various heuristic/metaheuristic modifications to tackle the problem (Hanum et al., 2019). VRPs have become popular in the academic literature, and have been applied in many applications such as logistics, transportation and supply chain management (Wichapa \& Khokhajaikiat, 2017). Although the VRPs are hard to solve, VRPs have been the heart of supply chain management and logistics. Most of the VRPs only consider one type of commodity. There are a lot of practical problems in which different types of commodities cannot be mixed together in the same compartment during transportation. An example of a VRP variant is the fuel delivery problem, which is a multi-compartment vehicle routing problem (MCVRP). The context of the MCVRP for fuel delivery is to design the route to deliver

* Corresponding author

E-mail address: wasana.chml@gmail.com (W. Chowmali) 
multiple fuels from a central depot to a petrol station, using a fleet of multi-compartment vehicles, with each compartment having different fuels that need to be kept separate. However, this problem can be divided into many categories. For example, MCVRP with a heterogeneous fleet of vehicles is an extension of MCVRP. That is, MCVRP with a heterogeneous fleet is like MCVRP, with the additional constraint that every vehicle must have various capacities of multiple commodities. The vehicle which is used in the MCVRP with a heterogeneous fleet for fuel delivery problem is usually composed of multi-compartments (see Fig.1), which are used to separate one fuel type from other fuel types.

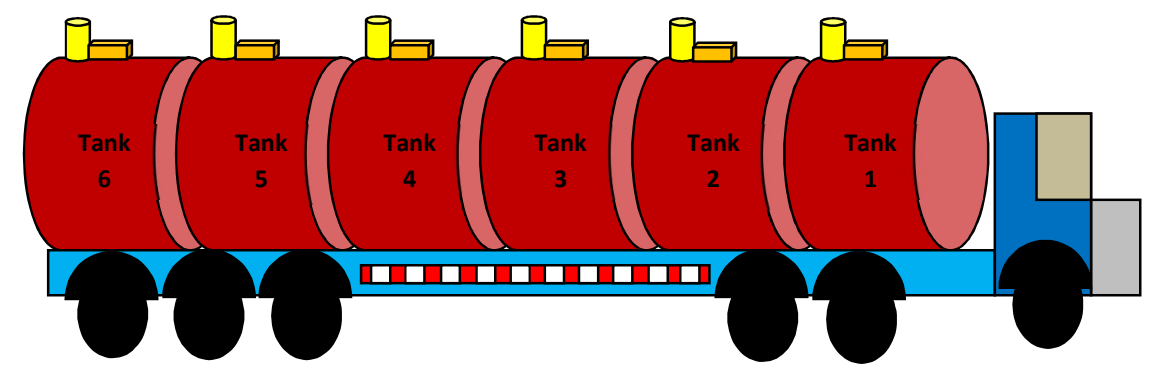

Fig.1. A fuel delivery vehicle with multi-compartments

Fig. 1 shows a multi-compartment vehicle which is used to deliver fuel, so different fuel types are not mixed. This makes the MCVRP with a heterogeneous fleet harder to solve using an exact method. All special characteristics occurring in the MCVRP with a heterogeneous fleet make the fuel-delivery problem more complex than the original VRP. These characters of the MCVRP with a heterogeneous fleet for fuel delivery are as follows: (1) one vehicle has multiple compartments, with each compartment having a different capacity; (2) vehicles have a variety of capacities; (3) Each vehicle compartment contains only one product; (4) each vehicle travels from a depot to a set of customer nodes and returns to a depot, and (5) the demand of each customer will be served by only one vehicle. These attributes make the fuel delivery problem, a variant of VRP, hard to solve.

The MCVRP with a heterogeneous fleet for fuel delivery aims to find a set of transport routes at a lowest operation cost, which is generated from the traveling cost and the vehicle cost, which depends on parameters including total driving distance and the number of vehicles which are used. In addition, like its particular case the VRP, the MCVRP with a heterogeneous fleet is an NP-hard problem. Hence, the MCVRP for this case turns into a very hard problem to solve. This is due to the fact that the optimal solution which is needed for the problem has to have the following conditions: (1) each vehicle has many compartments, each of which has different capacities; (2) there are a number of vehicles of various sizes, each of which has different vehicle costs; (3) there are many different vehicles, each of which may be chosen as the suitable vehicle for fuel delivery; (4) each vehicle's compartments can contain any fuel type, but they must not be mixed together. Also important is (5) other constraints that are the same as the original VRP, which is difficult to solve. Comparisons of the MCVRP for this paper with the original VRP and MCVRP are presented in Table 1. It is clear that the fleet of MCVRPs with a heterogeneous fleet is non-homogeneous, in the sense that the fleet consists of different types of vehicles with varying capacity for each product.

Table 1

Comparison of the VRPs

\begin{tabular}{cccc}
\hline Characteristic & Original VRP & MCVRP & MCVRP with a heterogeneous fleet of vehicles \\
\hline Vehicle & hetero/homo & homo & hetero \\
Compartment & single & multiple & multiple \\
Capacity of each compartment & single & single/multiple & multiple \\
Capacity of vehicle & single/various & single & multiple \\
\hline
\end{tabular}


From the literature review, the FJA, the work by Fisher and Jaikumar (1981) is one well-known heuristics that is often used for solving VRPs in various application areas. Certainly, in this case, the traditional algorithm needs to be adapted for solving the MCVRP with a heterogeneous fleet of vehicles for the fuel-delivery problem. Hence, a variant of FJA will be developed for solving the fuel-delivery problem for this case to obtain a suitable distribution network (a suitable framework of routes linking locations). The suitable distribution network for this case aims at a solution with a minimum total driving distance, using a minimum number of vehicles. The FJA has been modified in the following ways: (1) it formulates the new clustering-based model for selecting the seed nodes and seed vehicles, in which all customers are considered as candidate seed nodes, (2) it formulates the new GAP model to assign a customer to a vehicle and the customers assigned to a vehicle should be as close as possible to each other, and (3) after the customers are clustered, based on the TSP model, each cluster can be solved using LINGO software.

This research is categorized into six sections and organized as follows. Section 1, the Introduction, provides overall viewpoints, motivation and innovations of the article to the reader, and Section 2 is the literature review. In Section 3, we present the two phase heuristics for solving the proposed problem. Section 4 and Section 5 are devoted to the result and the conclusion of the article.

\section{Literature review}

\subsection{The fuel delivery problem}

In this section, the literature related to fuel delivery and distribution planning, with different types of fuels, and with multi-compartment trucks with different capacities, is reviewed about mathematical models and solving them with heuristics/meta-heuristics or exact methods. The solution approaches for solving the fuel delivery problem have been widely studied for over twenty years. For example, Benantar et al. (2016) proposed an efficient Tabu search algorithm for solving the MCVRP with time windows (MCVRPTW) for fuel distribution, while Chang et al. (2011) proposed optimization models for two possible cargo space layouts, and explored their characteristics with a Lagrangean heuristic. They generated a Set partitioning model, using a Branch and price algorithm to find the optimal solution for satisfying the orders, managing available resources, with the lowest total cost. In another technique, $\mathrm{Ng}$ et al. (2008) proposed a decision support system (DSS) combination with heuristic clustering and optimal routing to solve the multi-objective model for fuel delivery: a case study in Hong Kong. Surjandari et al. (2011) proposed the Tabu search algorithm for solving the petrol station replenishment problem. Carotenuto et al. (2015) proposed a Hybrid genetic algorithm for solving the periodic vehicle routing problem (PVRP) for fuel-oil distribution. Moreover, a decision support approach hierarchical planning system was developed for solving oil procurement planning (Kallestrup et al., 2014). For other problems of fuel distribution, Coelho and Laporte (2015) presented a model for the distribution of petrol products from storage depots to a set of petrol stations with uncertain demand, agreements for exchange of products with other oil companies, and contracts with carriers using the PVRP, and solved it with a Hybrid genetic algorithm. Some problems of fuel oil distribution have been proposed with decision support for oil purchase and distribution optimization using an oil purchase prediction and planning optimization model to solve it (Yu et al., 2016). Fallahi et al. (2008) proposed the Memetic algorithm and Tabu search for solving the MCVRP, while Benantar et al. (2019) proposed the Improved tabu search algorithm for solving the petrol-station replenishment problem with adjustable demands. Popović et al. (2012) developed a Variable neighborhood search (VNS) heuristic for solving a multiproduct multi-period Inventory Routing Problem (IRP) in fuel delivery. Prescott-Gagnon et al. (2014) defined the model of the IRP and used three meta-heuristics to address it, namely, a Tabu search algorithm, a Large neighborhood search heuristic and a Column generation heuristic. Vidović et al. (2014) proposed a mathematical model for the multi-product multi-period Inventory IRP; the problem was solved using a Variable neighborhood descent search. Another fuel delivery problem, called the replenishment problem model, has been proposed, defined in the multi-period station replenishment 
problem (MPSRP) model (Cornillier et al., 2008). Then these researchers developed a mathematical model for the petrol station replenishment problem with time windows (PSRPTW) with a single depot (Cornillier et al., 2012), and also continued to develop mathematical models of the multi-depot petrol station replenishment problem with time windows (MPSRPTW) (Cornillier et al., 2009), with all of the models solved by heuristics, while other researchers (Avella et al., 2004) proposed a heuristic and branch and price algorithm to solve a petrol replenishment problem with several tank-trucks of different types. Besides the several real-world applications of the MCVRPs in the context of fuel delivery, other real-world applications of the MCVRPs have been widely studied, as shown in the literature (Caramia \& Guerriero, 2010; De et al., 2018; Fallahi et al., 2008).

\subsection{Fisher and Jaikumar algorithm (FJA)}

Various ways have been proposed for solving VRPs in the literature (Casazza et al., 2018; GutierrezRodríguez et al., 2019; Gutierrez et al., 2018; Salavati-Khoshghalb et al., 2019). However, these can be divided into two categories, including heuristics/meta-heuristic methods and exact methods. Since no exact method can be guaranteed to find optimal tours within reasonable computing time when the number of nodes is large, the heuristic/meta-heuristic method has often been used in solving large problems of VRPs in the literature. Heuristic methods can be divided into Constructive heuristics and Two-phase heuristics, which are popular for solving VRPs. Some well-known Constructive heuristics are the Savings algorithm, Christofides algorithm, Matching based algorithm, Nearest Merger algorithm and Multi-route improvement heuristics. On the other hand, Two-phase heuristics are divided into two classes: (1) Cluster first-route second and (2) Route first-cluster second. In the first class, customers are assigned into the feasible cluster, and optimum routes are constructed for each cluster using the TSP model. In the second class, "a giant tour" is first built and then segmented into feasible vehicle routes. Some well-known heuristics of two-phase heuristics are the Sweep algorithm, FJA, Petal algorithm and Taillard's algorithm. The FJA (Fisher \& Jaikumar, 1981) is one of various Twophase algorithms, and is a well-known algorithm for solving the capacitated vehicle routing problem (CVRP). The general procedure of the FJA is comprised of four calculation steps (Baker \& Sheasby, 1999; Islam et al., 2015; Meindl \& Chopra, 2001) which are (1) it generates clusters with a geometric method partitioning each customer into each cone where the number of cones is equal to the number of vehicles, (2) seed nodes are selected from the cones and insertion cost is computed, (3) the generalized assignment problem model (GAP model) is employed to form the clusters and (4) the TSP model can be used to obtain the optimal travel cost. Undoubtedly, this algorithm has a major disadvantage: the efficiency of this algorithm is very sensitive to the location of the seed customers (Baker \& Sheasby, 1999; Islam et al., 2015; Meindl \& Chopra, 2001). Hence, finding the appropriate seed customers is one way to improve the quality of the algorithm to solve real world problems. In addition, there are currently no methods to confirm that any algorithm is the most effective way to solve the VRPs/MCVRPs, depending on the variant of each problem and individual preference. Although FJA is a well-known algorithm, the survey found that this method has not been applied to the MCVRP problem with a heterogeneous fleet for fuel delivery. These are the major reasons why FJA was selected as a suitable algorithm for solving MCVRP with a heterogeneous fleet of vehicles for fuel delivery in this case. Therefore, in this article, the FJA has been adapted for solving the fuel delivery problem. The proposed algorithm has been adapted in the following ways: (1) it formulates a new clusteringbased model for selecting the seed nodes and vehicles, in which all customers are considered as candidate seed nodes, (2) it formulates a new GAP model to assign a customer to a vehicle and the customers that are assigned to that vehicle are required to be as close to each other as possible, and (3)

after the customers are clustered, based on the TSP model, each cluster will be solved using LINGO software.

\section{Methodology}

This section presents a variant of FJA for solving the MCVRP with a heterogeneous fleet of vehicles for the fuel delivery problem. Details of the study framework are shown in Fig. 2. 


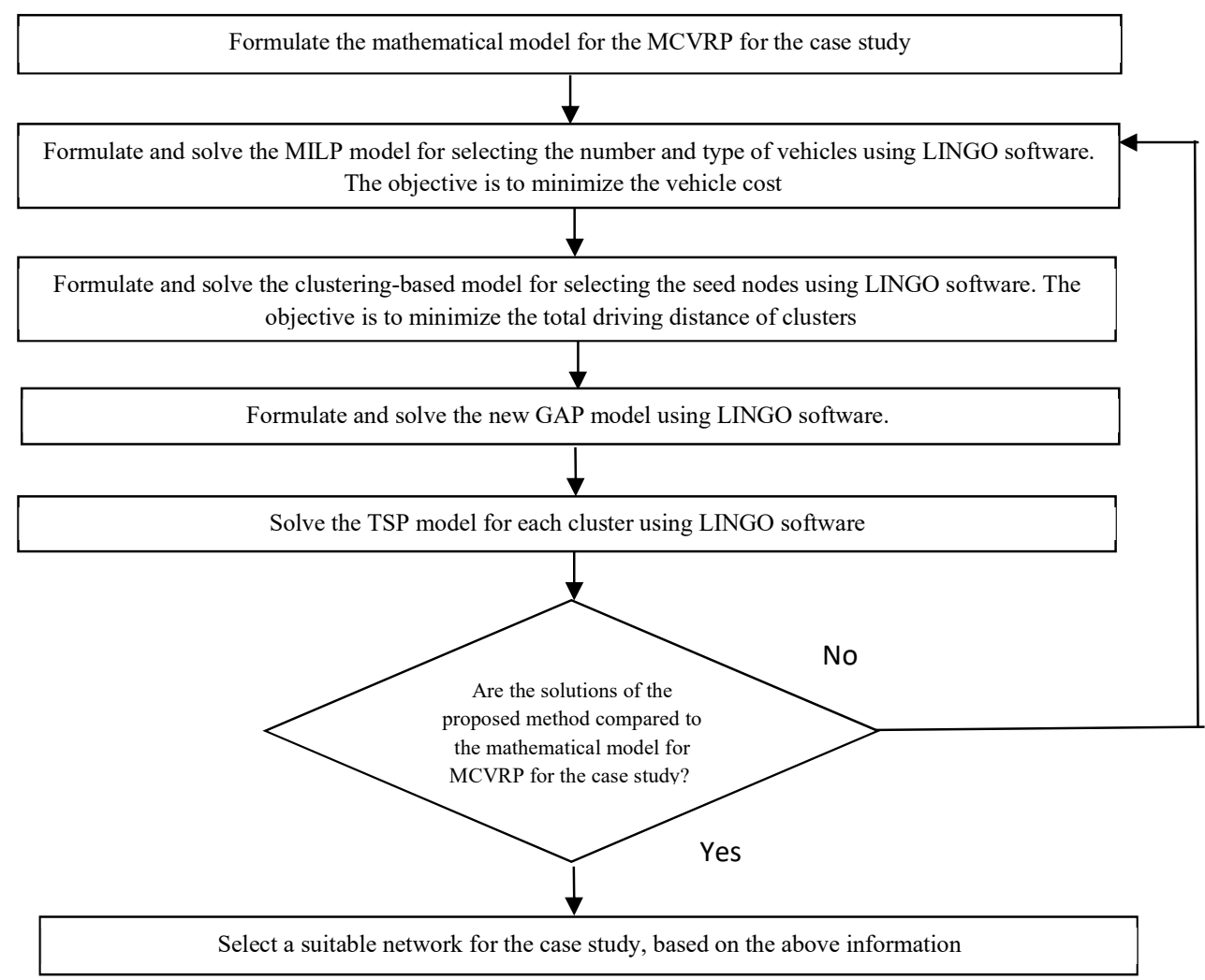

Fig.2. The study framework

\subsection{A MCVRP with a heterogeneous fleet of vehicles for the fuel delivery problem}

In this section, we present a mixed integer linear programming (MILP) model for fuel delivery. Since the MCVRP with a heterogeneous fleet problem is an extension of the MCVRP problem, the mathematical model for MCVRP in this case is a slight variation of the MCVRP model.

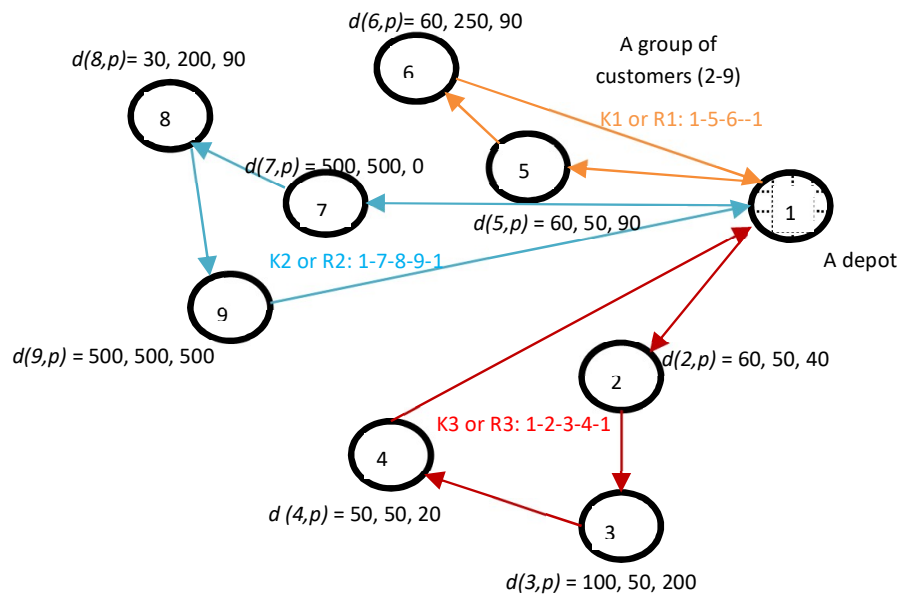

Fig. 3. A distribution network for fuel delivery

The MCVRP model with a heterogeneous fleet of vehicles can be formulated as an MILP model in the same way as the MCVRP model, where the constraints are adjusted such that different types of vehicles are allowed. Then the details of the mathematical model for MCVRP with a heterogeneous fleet for fuel delivery problem are shown in Fig.3. 


\section{Indices:}

The MCVRP model with a heterogeneous fleet for fuel delivery may be defined on a completely undirected network with a set of nodes $N=\{0,1,2, \ldots, n)$ including one depot (node 0 ) and a set $N^{\prime}$ of $n$ customers. Let $G=(N, A)$ be a complete graph where $N$ is the node set and $A$ is the $\operatorname{arc} \operatorname{set}$. $\operatorname{Arc}(i, j)$ $\in A$. $K$ is a set of multi-compartment vehicles that are available at the depot. $P$ is a set of fuel types.

Parameters: $d t_{i j}$ is the actual distance from node $i$ to node $j(\mathrm{~km}) . d_{j p}$ is the demand of the customer $j$ for fuel type $p$ (liter). $Q_{k p}$ is the capacity of vehicle $k$ for fuel type $p$ (liters), $Q_{k p}$ is determined by calculating the GAP model from section 3.3.3. $M L$ is a maximum route length.

\section{Decision variables:}

$X_{i j k}$ is a binary variable; $X_{i j k}=1$ if the node $i$ and node $j$ are linked by vehicle $k$;

$X_{i j k}=0$ otherwise.

$Y_{j k p}$ is a binary variable; $Y_{j k p}=1$ if the fuel type $p$ at node $j$ is serviced by vehicle $k$;

$Y_{j k p}=0$ otherwise.

\section{Objective function:}

$$
\min Z=\sum_{i \in N} \sum_{j \in N^{\prime}} \sum_{k \in K} d t_{i j} \cdot X_{i j k}
$$

subject to

$$
\begin{array}{lc}
\sum_{i \in N} X_{i j k} \leq 1, \quad \forall j \in N^{\prime}, \forall k \in K \\
\sum_{i \in N} X_{i j k}=\sum_{i \in N^{\prime}} X_{j i k}, & \forall j \in N^{\prime}, \forall k \in K \\
\sum_{i, i \in S} X_{i j k} \leq|S|-1, & \forall k \in K, \forall S \subseteq N^{\prime},|S| \geq 2 \\
Y_{j k p} \leq \sum_{i \in N} X_{i j k}, & \forall j \in N^{\prime}, \forall k \in K, \forall p \in P \\
\sum_{k \in K} Y_{j k p}=1, & \forall j \in N^{\prime}, \forall p \in P \\
\sum_{i \in N^{\prime}} d_{j p} \cdot Y_{j k p} \leq Q_{k p}, & \forall k \in K, \forall p \in P \\
\sum_{i, i \in N} d t_{i j} \cdot X_{i j k} \leq M L & \\
X_{i j k}, Y_{j k p} \in\{0,1\} &
\end{array}
$$

The objective function given by Eq. (1) represents the total driving distance of the transport routes to be minimized. Eq. (2) means that each customer $j$ may be visited at most once by each route. Eq. (3) means that if a multi-compartment vehicle enters customer $j$, it must leave it. Eq. (4) is a sub-tour elimination constraint. Eq. (5) means that if customer $j$ is not visited by vehicle $k, Y_{j k p}$ is equal to zero. Eq. (6) means that each customer $j$ with demand for fuel type $p$ is serviced by one single vehicle. Eq.n (7) means that the amount of each fuel cannot exceed its compartment capacity. Eq. (8) ensures that the route length cannot exceed the maximum route length. Eq. (9) means that variables $X, Y$ are binary.

\subsection{A MILP model for selecting the number and type of vehicles}

Due to the variety of the candidate vehicles, an MILP model for selecting the number and types of vehicles must be evaluated first, in order to minimize the vehicle cost for fuel delivery. Details of the model are shown below.

Indices: $j$ is a set of customers. $j=1,2,3, \ldots, J(J=20) . k$ is a set of candidate vehicles, $k=1,2,3, \ldots$, $K(K=5) . m$ is a set of the candidate compartments for each vehicle, $m=1,2,3, \ldots, M(M=7)$. $p$ is a set of the product types, $p=1,2,3, \ldots, P(P=3)$. 
Parameters: $v c_{k}$ is the vehicle cost of each vehicle $k$ (baht). $c v_{k}$ is the capacity of vehicle $k$ (liters). $d_{j p}$ is the demand for each product $p$ at petrol station $j$

\section{Decision variables:}

$X_{k m p}$ is a binary variable; $X_{k m p}=1$ if product type $p$ is serviced by the vehicle $k$ and compartment $m$; $X_{k m p}=0$ otherwise.

$Y_{j k}$ is a binary variable; $Y_{j k}=1$ if the customer $j$ is serviced by vehicle $k ; Y_{j k}=0$ otherwise.

$Z_{k}$ is a binary variable; $Z_{k}=1$ if the vehicle $\mathrm{k}$ is selected; $Z_{k}=0$ otherwise.

$W_{k m p}$ is the volume of the fuel $p$ which is contained in the compartment $m$ of the vehicle $k$.

Objective function:

$$
\min Z=\sum_{k-1}^{K} v c_{k} \cdot Z_{k}
$$

subject to:

$$
\begin{aligned}
& \sum_{p=1}^{P} X_{k m p} \leq 1, \quad \forall k, \forall m \\
& \sum_{k=1}^{K} Y_{j k}=1, \quad \forall j \\
& Y_{j k} \leq Z_{k}, \quad \forall j, \forall k \\
& X_{k m p} \leq \sum_{j=1}^{J} Y_{j k}, \quad \forall k, \forall m, \forall p \\
& W_{k m p} \leq c v_{k m} \cdot X_{k m p}, \quad \forall k, \forall m, \forall p \\
& \sum_{k=1}^{K} \sum_{m=1}^{M} W_{k m p}=\sum_{j=1}^{J} \sum_{k=1}^{K} d_{j p} \cdot Y_{j k}, \quad \forall p \\
& \sum_{m=1}^{M} W_{k m p}=\sum_{j=1}^{J} d_{j p} \cdot Y_{j k}, \quad \forall k, \forall p \\
& Q_{k p}=\sum_{m=1}^{M} W_{k m p}, \quad \forall k, \forall p \\
& X_{k m p}, Y_{j k}, Z_{k} \in\{0,1\}
\end{aligned}
$$

The objective function given by Eq. (10) represents the total vehicle cost for the selected vehicles to be minimized. Eq. (11) means that each compartment $m$ of the vehicle $k$ cannot contain more than one fuel type. Eq. (12) means that each customer $j$ is serviced by only one vehicle. Eq. (13) means that customer $j$ will be served by vehicle $k$ only when the vehicle $k$ is selected. Eq. (14) means that each compartment $m$ of the vehicle $k$ can contain product $p$ only when the customer $j$ is serviced by vehicle $k$. Eq. (15) means that the volume of each fuel cannot exceed its compartment capacity. Eq. (16) means that the total volume of each fuel type that is loaded in all vehicles is equal to the total demand of each fuel type. Eq. (17) means that the volume of each fuel type $p$ for each vehicle $k$ is equal to the demand of each fuel type $p$ for each customer $j$. Eq. (18) limits the capacity of each vehicle $k$ for each product $p$. Eq. (19) means that variables $X, Y$ and $\mathrm{Z}$ are binary.

\subsection{A variant of FJA for solving the MCVRP for fuel delivery}

In this section, the variant of FJA is proposed. Details of the variant of FJA are shown below.

- Formulate the new clustering-based model in order to choose the seed nodes and to assign a vehicle to each of the seeds, Eq. (20) to Eq. (28).

- Evaluate the insertion cost of each customer with respect to each seed, Eq. (29).

- For the new GAP model for solving the fuel delivery problem, Eq. (30) is the objective function and Eq. (11) to Eq. (19) are the constraints. The model can be calculated using LINGO software.

- TSP model for each cluster can be solved using LINGO software.

Details of each calculation step are shown below. 


\subsubsection{A new clustering-based model for seed selection}

Unlike the traditional seed selection of FJA, a new clustering-based model is developed for selecting the seed nodes. In this paper, the seed nodes are chosen using the new clustering-based model for which all customers are viewed as candidate seed nodes. Details of the proposed model are as follows.

Indices: $i$ is a set of candidate seed nodes, $i=1,2, \ldots, I(I=20), j$ is a set of customers, $j=1,2, \ldots, J$ $(J=20) . k$ is a set of vehicles, $k=1,2, \ldots, K(K=3) . K$ is determined using the MILP model for selecting the number and type of vehicles in Section 3.2. $m$ is a set of compartments of each vehicle. $m=1,2, \ldots$, $M(M=7) \cdot p$ is a set of products/fuels. $p=1,2, \ldots, P(P=3)$.

Parameters: $d t_{i j}$ is the actual distance matrix from seed node $i$ to customer $j . N V$ is the number of clusters $(N V=3) . d_{j p}$ is the demand for fuel $p$ for each customer $j . c v_{k}$ is the capacity of each vehicle $k$.

Variables:

$X_{i j}$ is a binary variable; $X_{i j}=1$ if the customer $j$ is serviced by seed node $i ; X_{i j}=0$ otherwise.

$Y_{i k}$ is a binary variable; $Y_{i k}=1$ if the vehicle $k$ is selected by seed node $i ; Y_{i k}=0$ otherwise.

$$
\begin{aligned}
& \min Z=\sum_{i=1}^{I} \sum_{j=1}^{J} d t_{i j} \cdot X_{i j} \\
& \sum_{i=1}^{I} X_{i j}=1, \quad \forall j \\
& \sum_{k=1}^{K} Y_{i k} \leq 1, \quad \forall i \\
& X_{i j} \leq \sum_{k=1}^{K} Y_{i k} ; \quad \forall i, \forall j \\
& \sum_{i=1}^{I} \sum_{k=1}^{K} Y_{i k}=N V ; \\
& \sum_{i=1}^{I} Y_{i k} \leq 1, \quad \forall k \\
& \sum_{i=1}^{J} \sum_{n=1}^{P} d_{j p} \cdot X_{i j} \leq \sum_{k=1}^{K} c v_{k} \cdot Y_{i k}, \quad \forall i \\
& X_{i j}, Y_{i k} \in\{0,1\}
\end{aligned}
$$

The objective function given by Eq. (20) represents the total driving distance, to be minimized. Eq. (21) means that each customer $j$ will be clustered into only a seed node $i$. Eq. (22) means that each seed node $i$ can select the number and type of vehicles that does not exceed one. Eq. (23) means that customer $\mathrm{j}$ will be served by seed node $i$ only when the vehicle $k$ is selected as seed vehicle at seed node $i$. Eq. (24) means that the number of clusters must not exceed a predetermined number. Eq. (25) means that each seed vehicle $k$ can select a number of seed nodes that does not exceed one. Eq. (26) means that each seed node $i$ with vehicle $k$ can support the products/fuels without exceeding its capacity. Eq. (27) means that variables $X$ and $Y$ are binary constraints. LINGO software can be employed to solve this model. After obtaining the initial seed solution from the above model, the final seed can be obtained using Eq. (28).

$$
a d t_{i}=d t_{o i}-d_{s i}, \quad i=1,2, \ldots, I
$$

where $a d t_{i}$ is the adjusted distance of the final seed for each candidate seed in each cluster (all customers in each cluster are considered as a new candidate. $d t_{o i}$ is the actual distance from a depot to a new candidate seed in each cluster. $d t_{s i}$ is the actual distance from a candidate seed to a depot. The final seed of each cluster is a candidate seed with the maximum value of the adjusted distance. 


\subsubsection{The insertion cost calculation}

After seed selection in Section 3.3.1, the insertion cost of customer $j$ is calculated, which is the cost of inserting that customer in the route going from seed customer to the depot. Then the customers are assigned to vehicles according to the increasing order of insertion cost. In this paper, the insertion cost of customer $j$ or $d_{j k}$ can be calculated using Eq. (29).

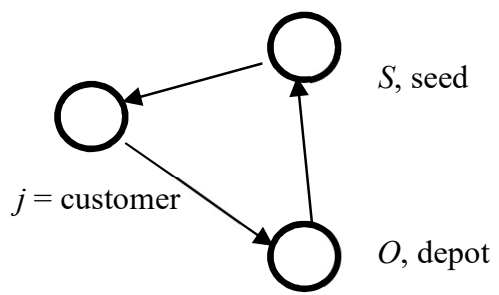

Fig.4. Demonstration of visiting a customer $j$

$$
d t_{j k}=d t_{s j}+d t_{j o}-d t_{o s}
$$

where $d t_{s j}$ is the actual distance from a seed to a customer. $d t_{j o}$ is the actual distance from a customer to a depot. $d t_{o s}$ is the actual distance from a depot to a seed.

\subsubsection{A new GAP model for assignment of customers}

In this section, a new GAP will be proposed in order to allocate the customers to seeds. The indices, parameters and variables are the same model as in section 3.2. However, the objective function has changed as follows.

$$
\min Z=\sum_{j=1}^{J} \sum_{k=1}^{K} d t_{j k} \cdot X_{j k}
$$

The objective function given by Eq. (33) is to minimize the total driving distance of all clusters. The constraints of this model are the same model as in section 3.2 including Eq. (11) to Eq. (19).

\subsubsection{A TSP model for optimum route generation}

In this section, the generation of each route for an individual vehicle is the final step to get the MCVRP solution with the clustered customer. The aim of this is to find the optimal transport route of a vehicle that represents the shortest path between all nodes in each cluster generated by the clustering model, in which each cluster is an individual traveling salesman problem (TSP) and LINGO software can be used to solve the TSP in this case. Details of the TSP model can be found in (Miller et al., 1960).

\section{Application example}

Since the competitive situation for business in Thailand is heating up, the various businesses must adjust their competitive strategies to reduce costs and increase customer service levels. Fuel delivery planning is one of the key success factors of this business, because it can reduce the transportation cost, which will make entrepreneurs more profitable. Thus, cost management, maintaining a low cost, can increase the efficiency and enhance the profits for entrepreneurs. In this research we introduce a case study of a petrol station, for which a retailer in fuel distribution transports fuel from a depot in the Central region of Thailand to petrol stations in the Northeast of Thailand. The distance between the depot and the petrol station is about 400 kilometers, with a transportation lead time of about 2 days per trip. The study was done in 20 petrol stations/customers $(C 1, C 2, \ldots, C 20)$ and a central depot $(D)$, see details in Fig. 5. In the current situation, the planning process for the company is based on experiment, without any effective information before assigning the trucks to travel to the depot, which directly affects the high transportation costs and also does not achieve customer requirements. Moreover, for fuel distribution, there are many restrictions that must be managed to be effective, such as truck fleet size, truck 
compartments and customer demand, so this research aims to find the optimal transport routes for fuel delivery, before the decision is made to release trucks, to minimize the costs for each trip (to minimize the total travel cost while using a minimum number of vehicles for fuel delivery). Details of each calculation step are shown in sections 4.1 and 4.2.

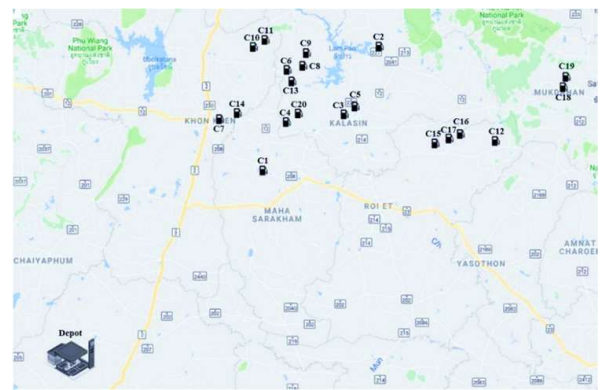

Fig. 5. The distribution network for the fuel delivery problem

\subsection{Select the number and type of vehicles}

The data for the analysis was collected as follows. The demands of each petrol station $\left(d_{j p}\right)$ are shown in Table 2. Let $v c_{1}, v c_{2}, v c_{3}, v c_{4}$ and $v c_{5}\left(v c_{k}\right)$ be 1705, 1675, 1675, 1600 and 1600 baht/trip respectively. The values of $c v_{1}, c v_{2}, c v_{3}, c v_{4}$ and $c v_{5}$ are shown in Table 3. After that, the LINGO software was used to solve this case using Eq. (10) to Eq. (19). The results are shown in Table 4.

Table 2

The demands for each fuel type of each petrol station

\begin{tabular}{|c|c|c|c|c|c|}
\hline ID & Name & $\begin{array}{c}\text { Demands }\left(\boldsymbol{d}_{\boldsymbol{j} \boldsymbol{p}}\right) \\
\text { (Diesel, Gas95, Gas91) }\end{array}$ & ID & Name & $\begin{array}{c}\text { Demands }\left(\boldsymbol{d}_{\boldsymbol{j} p}\right) \\
\text { (Diesel, Gas95, Gas91) }\end{array}$ \\
\hline$D$ & Depot (Saraburi) & $(0,0,0)$ & $C 11$ & Kranuan2 & $(5500,0,0)$ \\
\hline$C 1$ & Maha Sarakham1 & $(9000,0,0)$ & $C 12$ & Nong Phok & $(4000,0,0)$ \\
\hline$C 2$ & Somdet & $(14500,0,0)$ & C13 & Huai Mek2 & $(6000,500,1000)$ \\
\hline$C 3$ & Kalasin2 & $(6500,0,0)$ & $C 14$ & Chiang Yuen Maha Sarakham3 & $(4000,0,0)$ \\
\hline$C 4$ & Hua Na Khum1 & $(12000,0,0)$ & $C 15$ & Phon Thong2 & $(2000,2000,1000)$ \\
\hline$C 5$ & Phon Thong1 & $(5000,0,0)$ & C16 & Phon Thong3 & $(4000,0,0)$ \\
\hline C6 & Huai Mek1 & $(6000,0,0)$ & $C 17$ & Hua Na Khum2 & $(3500,5500,0)$ \\
\hline$C 7$ & Phra Lab & $(5500,0,0)$ & C18 & Mukdahan2 & $(4500,2000,500)$ \\
\hline$C 8$ & Nong Kung Si2 & $(4500,3000,0)$ & C19 & Non Tun Mukdahan3 & $(6000,0,0)$ \\
\hline$C 9$ & Nong Kung Si3 & $(4500,2500,500)$ & $C 20$ & Ban Kae & $(3,000,1,000,0)$ \\
\hline C10 & Kranuan 1 & $(4000,0,0)$ & & & \\
\hline
\end{tabular}

Table 3

The capacity for each candidate vehicle

\begin{tabular}{|c|c|c|c|c|c|c|c|c|}
\hline \multirow[b]{2}{*}{ Vehicle } & \multicolumn{8}{|c|}{ Component } \\
\hline & $m 1$ & $m 2$ & $m 3$ & $m 4$ & $m 5$ & $m 6$ & $m 7$ & Total \\
\hline$k 1$ & 9,000 & 6,000 & 6,000 & 6,000 & 6,000 & 6,000 & 8,000 & 47,000 \\
\hline$k 2$ & 9,000 & 8,000 & 7,000 & 7,000 & 7,000 & 7,000 & 0 & 45,000 \\
\hline$k 3$ & 9,000 & 8,000 & 7,000 & 7,000 & 7,000 & 7,000 & 0 & 45,000 \\
\hline$k 4$ & 8,000 & 6,000 & 4,000 & 4,000 & 4,000 & 6,000 & 8,000 & 40,000 \\
\hline$k 5$ & 8,000 & 6,000 & 4,000 & 4,000 & 4,000 & 6,000 & 8,000 & 40,000 \\
\hline
\end{tabular}

As seen in Table 4, the selected vehicles were $k 1 /$ vehicle $1\left(c v_{1}=47,000\right), k 2 /$ vehicle $2\left(c v_{2}=45,000\right)$ and $k 3 /$ vehicle $3\left(c v_{3}=45,000\right)$. Total vehicle cost $=1705+1675+1675=5055$ baht .

Table 4

The opened compartments of each selected vehicle for the case study

\begin{tabular}{|c|c|c|c|}
\hline \multirow[b]{2}{*}{ Results } & \multicolumn{3}{|c|}{ vehicle } \\
\hline & $k 1$ & $K 2$ & $k 3$ \\
\hline Opened compartments & $\begin{aligned} \operatorname{Diesel}\left(p_{1}\right) & =m 1, m 2, m 4, m 5, m 6 \\
\operatorname{Gas} 95\left(p_{2}\right) & =m 7 \\
\operatorname{Gas} 91\left(p_{3}\right) & =m 3\end{aligned}$ & $\begin{array}{l}\text { Diesel }\left(p_{l}\right)=m 1, m 2, m 3, m 4, m 5, m 6 \\
\text { Gas95 }\left(p_{2}\right)=0 \\
\operatorname{Gas} 91\left(p_{3}\right)=0\end{array}$ & $\begin{array}{l}\text { Diesel }\left(p_{1}\right)=m 2, m 3, m 4, \quad m 5, \\
m 6\end{array}$ \\
\hline$Q_{K P}$ (liter) & $\begin{array}{l}Q_{11}=33,000 \\
Q_{12}=8,000 Q_{13}=6,000 \\
\text { Total }=\mathbf{4 7 , 0 0 0}\end{array}$ & $\begin{array}{l}Q_{21}=45,000 \\
Q_{22}=0 Q_{23}=0 \\
\text { Total }=\mathbf{4 5 , 0 0 0}\end{array}$ & $\begin{array}{l}Q_{31}=36,000 \\
Q_{32}=9,000 Q_{33}=0 \\
\text { Total }=\mathbf{4 5 , 0 0 0}\end{array}$ \\
\hline
\end{tabular}




\subsection{Solve the MCVRP for this case using a variant of FJA}

After obtaining the suitable vehicles from Section 4.1, a variant of FJA was proposed. Details of the calculation steps are as follows:

\subsubsection{Choose the seed nodes using the new clustering-based model}

For choosing the seed nodes using Eq. (20) to Eq. (28), set $N V=3$. The demands $\left(d_{j p}\right)$ of each petrol station are shown in Table 2, and the capacity of each vehicle $k\left(c v_{k}\right)$ is shown in Table 3 . The number and type of vehicles in Table 4 were used for this calculation step. The actual distance matrix from the candidate seed node $i$ to the petrol station $j$ is shown in Table 5 as $d t_{i j}$. After that, the LINGO software was used to choose the seed nodes using Equations (20) to (27).

\section{Table 5}

The actual distance matrix from the candidate seed node $i$ to the petrol station $j$

\begin{tabular}{|c|c|c|c|c|c|c|c|c|c|c|c|c|c|c|c|c|c|c|c|c|c|}
\hline$I D$ & $D$ & $\mathrm{Cl}$ & $C 2$ & C3 & C4 & C5 & C6 & $C 7$ & $C 8$ & C9 & $\mathrm{ClO}$ & C11 & $\mathrm{Cl}$ & $\mathrm{Cl3}$ & C14 & C15 & $\mathrm{Cl6}$ & $\mathrm{Cl7}$ & C18 & $\mathrm{Cl9}$ & $\mathrm{C} 2 \mathrm{O}$ \\
\hline$D$ & 0 & 368 & 473 & 424.2 & 390 & 430 & 406 & 353 & 419.7 & 426.4 & 406 & 419 & 488 & 408 & 369 & 436.5 & 460 & 465 & 544 & 538 & 405 \\
\hline $\mathrm{Cl}$ & 368 & 0 & 116 & 67 & 58 & 72.6 & 77 & 50.1 & 85.7 & 92.4 & 95.6 & 109 & 135 & 71.3 & 50.6 & 96.5 & 120 & 54.7 & 194 & 188 & 53.1 \\
\hline$C 2$ & 473 & 116 & 0 & 53 & 92.3 & 46 & 66.2 & 75.3 & 54.5 & 61.2 & 91.1 & 104.6 & 96.4 & 65.1 & 114 & 53 & 76.5 & 88.9 & 116 & 110 & 83.9 \\
\hline C3 & 424.2 & 67 & 53 & 0 & 43.4 & 10.2 & 59 & 21.1 & 65.3 & 72 & 79 & 92.5 & 95.3 & 52.9 & 65.3 & 48.2 & 71.7 & 53.7 & 147.2 & 141.2 & 31.8 \\
\hline C4 & 390 & 58 & 92.3 & 43.4 & 0 & 49 & 30.2 & 28.3 & 51.2 & 57.9 & 55 & 68.5 & 122 & 24.4 & 30 & 74.6 & 98.1 & 3.3 & 182 & 176 & 13.2 \\
\hline C5 & 430 & 72.6 & 46 & 10.2 & 49 & 0 & 64.6 & 31.1 & 60 & 66.7 & 89.4 & 102.9 & 99.5 & 58.8 & 70.8 & 52.4 & 75.9 & 45.7 & 140 & 134 & 40.6 \\
\hline C6 & 406 & 77 & 66.2 & 59 & 30.2 & 64.6 & 0 & 44 & 29 & 35.7 & 24.8 & 38.3 & 137 & 5.8 & 41.1 & 90.5 & 114 & 34 & 176 & 170 & 25 \\
\hline$C 7$ & 353 & 50.1 & 75.3 & 21.1 & 28.3 & 31.1 & 44 & 0 & 65 & 71.7 & 68.8 & 82.3 & 93.3 & 38.2 & 50.2 & 46.3 & 69.8 & 25.1 & 154 & 148 & 20 \\
\hline C8 & 419.7 & 85.7 & 54.5 & 65.3 & 51.2 & 60 & 29 & 65 & 0 & 6.7 & 50.2 & 63.7 & 160.7 & 26.8 & 70.7 & 111.2 & 134.7 & 55 & 164.7 & 158.7 & 46 \\
\hline C9 & 426.4 & 92.4 & 61.2 & 72 & 57.9 & 66.7 & 35.7 & 71.7 & 6.7 & 0 & 56.9 & 70.4 & 167.4 & 33.5 & 77.4 & 117.9 & 141.4 & 61.7 & 171.4 & 165.4 & 52.7 \\
\hline $\mathrm{C} 10$ & 406 & 95.6 & 91.1 & 79 & 55 & 89.4 & 24.8 & 68.8 & 50.2 & 56.9 & 0 & 13.5 & 162 & 30.6 & 51.6 & 115.5 & 139 & 58.8 & 194 & 188 & 49.8 \\
\hline $\mathrm{Cll}$ & 419 & 109 & 104.6 & 92.5 & 68.5 & 102.9 & 38.3 & 82.3 & 63.7 & 70.4 & 13.5 & 0 & 175.5 & 44.1 & 65.1 & 129 & 152.5 & 72.3 & 207.5 & 201.5 & 63.3 \\
\hline $\mathrm{Cl} 2$ & 488 & 135 & 96.4 & 95.3 & 122 & 99.5 & 137 & 93.3 & 160.7 & 167.4 & 162 & 175.5 & 0 & 132 & 144 & 8.1 & 31.6 & 118 & 77.1 & 71.1 & 113 \\
\hline $\mathrm{Cl} 13$ & 408 & 71.3 & 65.1 & 52.9 & 24.4 & 58.8 & 5.8 & 38.2 & 26.8 & 33.5 & 30.6 & 44.1 & 132 & 0 & 43.9 & 84.5 & 108 & 28.3 & 175 & 169 & 19.4 \\
\hline $\mathrm{Cl} 14$ & 369 & 50.6 & 114 & 65.3 & 30 & 70.8 & 41.1 & 50.2 & 70.7 & 77.4 & 51.6 & 65.1 & 144 & 43.9 & 0 & 95.5 & 119 & 26.8 & 204 & 198 & 52.8 \\
\hline $\mathrm{C} 15$ & 436.5 & 96.5 & 53 & 48.2 & 74.6 & 52.4 & 90.5 & 46.3 & 111.2 & 117.9 & 115.5 & 129 & 8.1 & 84.5 & 95.5 & 0 & 23.5 & 71.4 & 63.5 & 57.5 & 84.5 \\
\hline C16 & 460 & 120 & 76.5 & 71.7 & 98.1 & 75.9 & 114 & 69.8 & 134.7 & 141.4 & 139 & 152.5 & 31.6 & 108 & 119 & 23.5 & 0 & 94.9 & 87 & 81 & 108 \\
\hline $\mathrm{C} 17$ & 465 & 54.7 & 88.9 & 53.7 & 3.3 & 45.7 & 34 & 25.1 & 55 & 61.7 & 58.8 & 72.3 & 118 & 28.3 & 26.8 & 71.4 & 94.9 & 0 & 179 & 173 & 20.3 \\
\hline $\mathrm{C} 18$ & 544 & 194 & 116 & 147.2 & 182 & 140 & 176 & 154 & 164.7 & 171.4 & 194 & 207.5 & 77.1 & 175 & 204 & 63.5 & 87 & 179 & 0 & 6 & 173 \\
\hline $\mathrm{Cl} 19$ & 538 & 188 & 110 & 141.2 & 176 & 134 & 170 & 148 & 158.7 & 165.4 & 188 & 201.5 & 71.1 & 169 & 198 & 57.5 & 81 & 173 & 6 & 0 & 167 \\
\hline C2O & 405 & 53.1 & 83.9 & 31.8 & 13.2 & 40.6 & 25 & 20 & 46 & 52.7 & 49.8 & 63.3 & 113 & 19.4 & 52.8 & 84.5 & 108 & 20.3 & 173 & 167 & 0 \\
\hline
\end{tabular}

Finally, after calculation using Eq. (20) to Eq. (28), the results obtained show that the seed nodes were $C_{15}$ with $k 1\left(c v_{1}=47,000\right), C_{17}$ with vehicle $k 2\left(c v_{2}=45,000\right)$ and $C_{6}$ with vehicle $k 3\left(c v_{3}=45,000\right)$. After that, the final seed nodes were obtained using Equation (28), the new seed nodes were $C_{12}$ with $k 1\left(c v_{1}=47,000\right), C_{17}$ with vehicle $k 2\left(c v_{2}=45,000\right)$ and $C_{6}$ with vehicle $k 3\left(c v_{3}=45,000\right)$.

\subsubsection{Evaluate the insertion cost of each customer with respect to each seed}

The insertion cost of each customer with respect to each seed $\left(d_{j k}\right)$ was evaluated using Equation (29), and the results are shown in Table 6.

Table 6

The insertion cost of each customer with respect to each seed

\begin{tabular}{|c|c|c|c|c|c|c|c|}
\hline$I D$ & $C 12(k=1)$ & $C 17(k=2)$ & C6 $(k=3)$ & $I D$ & $C 12(k=1)$ & $C 17(k=2)$ & $C 6(k=3)$ \\
\hline$C 1$ & 15 & -42.3 & 39 & $C 11$ & 106.5 & 26.3 & 51.3 \\
\hline$C 2$ & 81.4 & 96.9 & 133.2 & C12 & 0 & 141 & 219 \\
\hline C3 & 31.5 & 12.9 & 77.2 & C13 & 52 & -28.7 & 7.8 \\
\hline C4 & 24 & -71.7 & 14.2 & C14 & 25 & -69.2 & 4.1 \\
\hline$C 5$ & 41.5 & 10.7 & 88.6 & C15 & -43.4 & 42.9 & 121 \\
\hline C6 & 55 & -25 & 0 & C16 & 3.6 & 89.9 & 168 \\
\hline$C 7$ & -41.7 & -86.9 & -9 & C17 & 95 & 0 & 93 \\
\hline$C 8$ & 92.4 & 9.7 & 42.7 & C18 & 133.1 & 258 & 314 \\
\hline C9 & 105.8 & 23.1 & 56.1 & $C 19$ & 121.1 & 246 & 302 \\
\hline$C 10$ & 80 & -0.2 & 24.8 & $C 20$ & 30 & -39.7 & 24 \\
\hline
\end{tabular}




\subsubsection{Assign the customers to seed vehicles.}

The assignment of customers to seed vehicles was made using Equation (33) and Equations (12) to (22) for the constraints, and the results are shown in Table 7.

\section{Table 7}

The results of the GAP model for this case

\begin{tabular}{|c|c|c|c|}
\hline \multirow[b]{2}{*}{ Results } & \multicolumn{3}{|c|}{ Clusters } \\
\hline & k1 (C15) & $K 2(C 17)$ & $k 3(C 6)$ \\
\hline $\begin{array}{c}\text { Opened } \\
\text { compartments }\end{array}$ & $\begin{array}{l}\text { Diesel }\left(p_{1}\right)=m 1, m 3, m 4, m 5, m 6 \text { Gas95 }\left(p_{2}\right)=m 7 \\
\text { Gas91 }\left(p_{3}\right)=m 2\end{array}$ & $\begin{array}{l}\text { Diesel }\left(p_{1}\right)=m 2, m 3, m 4, m 5, m 6 \\
\text { Gas95 }\left(p_{2}\right)=m 1 \text { Gas91 }\left(p_{3}\right)=0\end{array}$ & $\begin{array}{l}\text { Diesel }\left(p_{1}\right)=m 1, m 2, m 3, m 4 \\
m 5, m 6 \operatorname{Gas} 95\left(p_{2}\right)=0 \\
\operatorname{Gas} 91\left(p_{3}\right)=0\end{array}$ \\
\hline Assigned customers & $\mathrm{C} 5, \mathrm{C} 9, \mathrm{C} 12, \mathrm{C} 13, \mathrm{C15}, \mathrm{C} 16, \mathrm{C} 18, \mathrm{C} 2 \mathrm{O}$ & $C 3, C 4, C 7, C 8, C 14, C 17$ & $C 1, C 2, C 6, C 10, C 11, C 19$ \\
\hline $\begin{array}{l}\text { Volume for each } \\
\text { vehicle (liter) }\end{array}$ & $\begin{array}{l}44,000(m 1=9000 \\
m 2=3000, m 3=6000, m 4=6000, m 5=6000 \\
m 6=6000, m 7=8000)\end{array}$ & $\begin{array}{l}44,500(m 1=8500, \\
m 2=8000, m 3=7000, m 4= \\
7000, m 5=7000, m 6=7000\end{array}$ & $\begin{array}{l}45,000(m 1=9000 \\
m 2=8000, m 3=7000, m 4= \\
7000, m 5=7000, m 6=7000\end{array}$ \\
\hline
\end{tabular}

\subsubsection{Generate the optimum routes using the TSP model}

The shortest path between all nodes in each cluster was generated using the TSP model. For this case, LINGO software can be used to solve the TSP model as in the literature, and the results are shown in Table 8.

\section{Table 8}

The results of the TSP model for this case

\begin{tabular}{|c|c|c|c|}
\hline \multirow[b]{2}{*}{ Results } & \multicolumn{3}{|c|}{ Clusters } \\
\hline & $k 1(C 15)$ & $k 3(C 17)$ & $k 2(C 6)$ \\
\hline Assigned customers & $\mathrm{C} 5, \mathrm{C} 9, \mathrm{C} 12, \mathrm{C} 13, \mathrm{C} 15, \mathrm{C} 16, \mathrm{C} 18, \mathrm{C} 2 \mathrm{O}$ & $\mathrm{C} 3, \mathrm{C4}, \mathrm{C} 7, \mathrm{C} 8, \mathrm{C14}, \mathrm{C} 17$ & $C 1, C 2, C 6, C 10, C 11, C 19$ \\
\hline The sequence of & $\begin{array}{l}D-C 16-C 12-C 18-C 15-C 5-C 20-C 13-C 9-D \\
\text { Distance for the cluster }=12045 \mathrm{~km}\end{array}$ & $\begin{array}{l}D-C 7-C 3-C 8-C 4-C 17-C 14-D \\
\text { Distance for the cluster }=889.7 \mathrm{~km}\end{array}$ & $\begin{array}{l}D-C 11-C 10-C 6-C 2-C 19-C 1-D \\
\text { Distance for the cluster }=11895 \mathrm{~km}\end{array}$ \\
\hline Total distance & $1204.5+889.7+1189.5=3,284 \mathrm{~km}$ & & \\
\hline
\end{tabular}

The results obtained for the proposed algorithm were compared with computational results using LINGO software based on the MCVRP model in section 3.1. The experimentation was performed on a computer with the following characteristics: Intel ${ }^{\circledR}$ Core $^{\mathrm{TM}}$ i5-4210U processor Dual-core at 1.70 GHz with 8 GB of RAM, and Windows 8.1 operating system. The comparison of solutions is shown in Table 9.

Table 9

Comparison of solutions using LINGO software and the proposed heuristic

\begin{tabular}{|c|c|c|c|c|c|c|c|}
\hline \multirow[b]{2}{*}{ Data set } & \multirow{2}{*}{$\begin{array}{c}\text { Number of } \\
\text { petrol } \\
\text { stations }\end{array}$} & \multicolumn{3}{|c|}{$\begin{array}{l}\text { LINGO software based on the mathematical model in } \\
\text { Section } 3.1\end{array}$} & \multicolumn{3}{|c|}{ Proposed heuristic } \\
\hline & & $\begin{array}{c}\text { Number and type } \\
\text { of vehicles } \\
(\mathrm{NV}) \\
\end{array}$ & $\begin{array}{c}\text { Total } \\
\text { distance } \\
\text { (TD) } \\
\end{array}$ & $\begin{array}{c}\text { Computational } \\
\text { times } \\
(\mathrm{hh}, \mathrm{mm}, \mathrm{ss}) \\
\end{array}$ & $\begin{array}{c}\text { Number and } \\
\text { type of vehicles } \\
(\mathrm{NV})\end{array}$ & $\begin{array}{c}\text { Total } \\
\text { distance } \\
\text { (TD) } \\
\end{array}$ & Deviation \\
\hline Problem 1 & 5 & $1(k 1)$ & 973 & $00: 00: 01$ & 1 & 973 & $0 \%$ \\
\hline Problem 2 & 10 & $2(k 3$ and $k 5)$ & 1836.5 & $00: 00: 26$ & $2(k 3$ and $k 5)$ & 1839 & $+0.14 \%$ \\
\hline Problem 3 & 15 & $3(k 3, k 4, k 5)$ & $2774.3^{*}$ & 168:00:00 & $3(k 3, k 4, k 5)$ & 2,780 & $+0.20 \%$ \\
\hline Actual problem & 20 & $3(k 1, k 2, k 3)$ & $3,292.1 *$ & 168:00:00 & $3(k 1, k 2, k 3)$ & 3,284 & $-0.24 \%$ \\
\hline
\end{tabular}

As seen in Table 8, the computational results show that the optimal solutions for the small size problem $(\mathrm{N}=5)$ were achieved by using LINGO software and the proposed heuristic, and the computational results using the proposed heuristic for $\mathrm{N}=10$ and $\mathrm{N}=15$ were slightly different from the optimal solution. In addition, the computational results using the proposed heuristic for $\mathrm{N}=20$ (the actual case) was better than the best known solutions at computational times of 168 hours using LINGO software. These are reasons why the proposed heuristic must be used for this case. This paper has provided realworld applications and additional insights for research, and it can guide scholars to establish a novel heuristic for solving the MCVRP for the fuel delivery problem which is an NP-hard problem. The proposed heuristic is flexible and applicable for solving VRPs in real-world situations. Therefore, it is 
believed that the proposed heuristic is a suitable tool for solving the MCVRP in this case study. In particular, it is believed that the proposed algorithm can be applied to tackle other VRPs in real-world situations.

\section{Conclusions}

In this paper, we have investigated a clustering based MCVRP solving method in which a variant of FJA was considered for clustering the nodes, and the TSP model has been employed for finding the optimal routes of individual vehicles. The proposed heuristic was tested with a case study comprising twenty petrol stations in Northeastern Thailand. We first utilized an MILP model to evaluate the number and type of vehicles. Secondly, the new clustering-based model was developed for selecting the seed nodes, where all customer nodes can be considered as candidates. After that, the new GAP model was formulated to allocate the customers into each cluster. Finally, the TSP model for each cluster was solved to minimize the total driving distance. The numerical results show the effectiveness of the proposed heuristic. The obtained results show that vehicle $k 1$, vehicle $k 2$ and vehicle $k 3$ were chosen as suitable vehicles for fuel delivery with lowest vehicle cost $(5,050 \mathrm{baht})$, minimum number of vehicles $(K=3)$ and lowest total distance $(3,284 \mathrm{~km})$ which are achieved using the proposed heuristic. The proposed variant FJA is effective, and it is useful and applicable for scholars to find a new way for solving the MCVRP with a heterogeneous fleet of vehicles, which differs from other heuristics in the literature. In particular, we believe that a variant of FJA can be applied to address other VRPs in realworld situations. For future research, application of the proposed heuristic should be tested with more cases of MCVRP to further enhance the validity of the research output. Also, for practical VRPs in which an exact solution cannot be found, the proposed heuristic can be applied.

\section{Acknowledgement}

The authors would like to thank the Graduate School, Khon Kaen University for financial support for this research under the grant number 582JT201.

\section{References}

Avella, P., Boccia, M., \& Sforza, A. (2004). Solving a fuel delivery problem by heuristic and exact approaches. European Journal of Operational Research, 152(1), 170-179.

Baker, B. M., \& Sheasby, J. (1999). Extensions to the generalised assignment heuristic for vehicle routing. European Journal of Operational Research, 119(1), 147-157.

Benantar, A., Ouafi, R., \& Boukachour, J. (2016). A petrol station replenishment problem: new variant and formulation. Logistics Research, 9(1), 6.

Benantar, A., Ouafi, R., \& Boukachour, J. (2019). An improved tabu search algorithm for the petrol-station replenishment problem with adjustable demands. INFOR: Information Systems and Operational Research, $1-21 .$.

Caramia, M., \& Guerriero, F. (2010). A heuristic approach for the truck and trailer routing problem. Journal of the Operational Research Society, 61(7), 1168-1180.

Carotenuto, P., Giordani, S., Massari, S., \& Vagaggini, F. (2015). Periodic capacitated vehicle routing for retail distribution of fuel oils. Transportation Research Procedia, 10, 735-744..

Casazza, M., Ceselli, A., \& Calvo, R. W. (2018). A branch and price approach for the Split Pickup and Split Delivery VRP. Electronic Notes in Discrete Mathematics, 69, 189-196.

Chang, M. H., Cho, S., Kang, H. G., Yun, S. H., Song, K. M., Kim, D., \& Chung, H. (2011). Process simulation for fuel delivery from storage and delivery system in fusion power plant. Fusion Engineering and Design, 86(9-11), 2200-2203..

Chokanat, P., Pitakaso, R., \& Sethanan, K. (2019). Methodology to Solve a Special Case of the Vehicle Routing Problem: A Case Study in the Raw Milk Transportation System. AgriEngineering, 1(1), 75-93.

Coelho, L. C., \& Laporte, G. (2015). Classification, models and exact algorithms for multi-compartment delivery problems. European Journal of Operational Research, 242(3), 854-864.

Cornillier, F., Boctor, F. F., Laporte, G., \& Renaud, J. (2008). A heuristic for the multi-period petrol station replenishment problem. European Journal of Operational Research, 191(2), 295-305. 
Cornillier, F., Boctor, F., \& Renaud, J. (2012). Heuristics for the multi-depot petrol station replenishment problem with time windows. European Journal of Operational Research, 220(2), 361-369.

Cornillier, F., Laporte, G., Boctor, F. F., \& Renaud, J. (2009). The petrol station replenishment problem with time windows. Computers \& Operations Research, 36(3), 919-935..

De, A., Pratap, S., Kumar, A., \& Tiwari, M. K. (2018). A hybrid dynamic berth allocation planning problem with fuel costs considerations for container terminal port using chemical reaction optimization approach. Annals of Operations Research, 1-29.

El Fallahi, A., Prins, C., \& Calvo, R. W. (2008). A memetic algorithm and a tabu search for the multicompartment vehicle routing problem. Computers \& Operations Research, 35(5), 1725-1741..

Fisher, M. L., \& Jaikumar, R. (1981). A generalized assignment heuristic for vehicle routing. Networks, 11(2), 109-124.

Gutierrez-Rodríguez, A. E., Conant-Pablos, S. E., Ortiz-Bayliss, J. C., \& Terashima-Marín, H. (2019). Selecting meta-heuristics for solving vehicle routing problems with time windows via meta-learning. Expert Systems with Applications, 118, 470-481.

Gutierrez, A., Dieulle, L., Labadie, N., \& Velasco, N. (2018). A multi-population algorithm to solve the VRP with stochastic service and travel times. Computers \& Industrial Engineering, 125, 144-156.

Hanum, F., Hadi, M., Aman, A., \& Bakhtiar, T. (2019). Vehicle routing problems in rice-for-the-poor distribution. Decision Science Letters, 8(3), 323-338.

Islam, M., Ghosh, S., \& Rahman, M. (2015). Solving Capacitated Vehicle Routing Problem by Using Heuristic Approaches: A Case Study. Journal of Modern Science and Technology, 3(1), 135-146.

Kallestrup, K. B., Lynge, L. H., Akkerman, R., \& Oddsdottir, T. A. (2014). Decision support in hierarchical planning systems: The case of procurement planning in oil refining industries. Decision Support Systems, 68, 49-63.

Chopra, S., \& Meindl, P. (2007). Supply chain management. Strategy, planning \& operation. In Das summa summarum des management (pp. 265-275). Gabler.

Miller, C. E., Tucker, A. W., \& Zemlin, R. A. (1960). Integer programming formulation of traveling salesman problems. Journal of the ACM (JACM), 7(4), 326-329.

Ng, W. L., Leung, S. C. H., Lam, J. K. P., \& Pan, S. W. (2008). Petrol delivery tanker assignment and routing: a case study in Hong Kong. Journal of the Operational Research Society, 59(9), 1191-1200.

Popović, D., Vidović, M., \& Radivojević, G. (2012). Variable neighborhood search heuristic for the inventory routing problem in fuel delivery. Expert Systems with Applications, 39(18), 13390-13398.

Prescott-Gagnon, E., Desaulniers, G., \& Rousseau, L. M. (2014). Heuristics for an oil delivery vehicle routing problem. Flexible Services and Manufacturing Journal, 26(4), 516-539.

Salavati-Khoshghalb, M., Gendreau, M., Jabali, O., \& Rei, W. (2019). An exact algorithm to solve the vehicle routing problem with stochastic demands under an optimal restocking policy. European Journal of Operational Research, 273(1), 175-189.

Surjandari, I., Rachman, A., Dianawati, F., \& Wibowo, R. P. (2011). Petrol delivery assignment with multiproduct, multi-depot, split deliveries and time windows. International Journal of Modeling and Optimization, 1(5), 375.

Vidović, M., Popović, D., \& Ratković, B. (2014). Mixed integer and heuristics model for the inventory routing problem in fuel delivery. International Journal of Production Economics, 147, 593-604.

Wichapa, N., \& Khokhajaikiat, P. (2017). Using the hybrid fuzzy goal programming model and hybrid genetic algorithm to solve a multi-objective location routing problem for infectious waste disposal. Journal of Industrial Engineering and Management, 10(5), 853-886.

Wichapa, N., \& Khokhajaikiat, P. (2018). Solving a multi-objective location routing problem for infectious waste disposal using hybrid goal programming and hybrid genetic algorithm. International Journal of Industrial Engineering Computations, 9(1), 75-98.

Yu, L., Yang, Z., \& Tang, L. (2016). Prediction-based multi-objective optimization for oil purchasing and distribution with the NSGA-II algorithm. International Journal of Information Technology \& Decision Making, 15(02), 423-451.

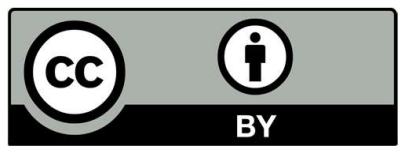

(C) 2020 by the authors; licensee Growing Science, Canada. This is an open access article distributed under the terms and conditions of the Creative Commons Attribution (CC-BY) license (http://creativecommons.org/licenses/by/4.0/). 